ARTICLE

\title{
High Performance Parallel Monte Carlo Transport Computations for ITER Fusion Neutronics Applications
}

\author{
Arkady SERIKOV*, Ulrich FISCHER and Dennis GROSSE \\ Karlsruhe Institute of Technology (KIT), Hermann-von-Helmholtz-Platz 1, \\ 76344 Eggenstein-Leopoldshafen, Germany
}

\begin{abstract}
Large scale neutronics calculations for radiation safety and machine reliability are required to support design activities for the ITER fusion reactor which is currently in phase of construction. Its large size and complexity of diagnostics, control and heating systems and ports, and also channel penetrations inside the thick blanket shielding surrounding the $14 \mathrm{MeV}$ D-T neutron source are essential challenges for neutronics calculations. In the ITER tokamak geometry, the Monte Carlo (MC) method is the preferred one for radiation transport calculations. Due to the independence of particle histories, their tracks can be processed in parallel. Parallel computations on high performance cluster computers substantially increase number of sampled particles and therefore allow reaching the desired statistical precision of the MC results using the MCNP5 code. The MCNP5 parallel performance was assessed on the HPC-FF supercomputer. Use of CAD-based approach with high spatial resolution improves systematic adequacy of the MC geometry modeling. These achievements are demonstrated on radiation transport calculations for designing the Blanket Shield Module and Auxiliary Shield of the ITER Electron Cyclotron Heating (ECH) upper launcher. The spatial distributions of nuclear heating were analysed by using the graphical representation of the MCNP5 mesh-tally results in 2D and 3D plots.
\end{abstract}

KEYWORDS: Monte Carlo, MCNP5 parallel computations, JUROPA/HPC-FF supercomputer, ITER, fusion neutronics, Electron Cyclotron Heating (ECH) upper launcher, CAD-based radiation transport, 3D mesh-tally visualization with VisIt

\section{Introduction}

The challenging problems of neutronic calculations in designing the Electron Cyclotron Heating (ECH) launcher to be installed in the upper port of International Thermonuclear Experimental Reactor (ITER) have been solved by employing the Monte Carlo MCNP5 code $^{1)}$ running in Message Passing Interface (MPI) parallel mode on high performance computer clusters. The methodological approach employed to tackle the neutronic analyses was based on the use of both standard MCNP5 variance reduction techniques (VRTs) and mesh-tally mapping of the results, as well as newly developed at KIT (former FZK) program interface McCad $^{2)}$ allowing automatic conversion of CAD models into the MCNP geometry representation and vice versa. The McCad interface has been successfully applied for modeling of several fusion neutronics applications. ${ }^{3)}$ In designing of the ITER components, this CAD-based modeling and MCNP5 mesh tally capability are inevitable for resolving heterogeneous neutronic effects. The ITER-specific fusion neutronics is characterized by radiation deep-penetration with total neutron flux attenuation by 8 orders of magnitude from the plasma to the cryostat. The ECH launcher to be inserted in the ITER upper port extends by $5 \mathrm{~m}$ from the plasma first wall (FW) to the vacuum vessel connective flange at the rear side close to cryostat. The current design of the Qua-

*Corresponding author, E-mail:arkady.serikov@kit.edu

(c) 2011 Atomic Energy Society of Japan, All Rights Reserved.
si-Optical (QO) ECH launcher is shown in Fig. 1. Some aspects of its nuclear-safety-related and shielding analyses have been considered in Reference 4, and this paper addresses the problems which require heterogeneous calculations utilizing high performance computational resources. The parts of the ECH launcher are analysed in this work include Blanket Shield Module (BSM) and Auxiliary Shield shown in Fig. 1 together with its other structures which serve for realization of the launcher' aim, which is to control Magneto - Hydro - Dynamic (MHD) instabilities in the ITER plasma. This is fulfilled by precisely focusing of millimeter waves (mm-waves) at $170 \mathrm{GHz}$ and injecting them onto the plasma magnetic surfaces by means of the launcher's Quasi-Optical (QO) mm-wave system. ${ }^{5)}$ To provide the mm-wave beam injection shown in Fig. 1, the launcher has to have an opening to plasma. This requires the arrangement of shielding blocks inside the launcher without interfering with the propagation of the mm-wave beams. The beams are directed from the launcher's back-end along rows of 8 wave-guide channels and void space of the QO system shown in Fig. 1.

In this work, the MC radiation transport computations were performed with the Alite standard ITER model ${ }^{6)}$ representing a $40^{\circ}$ torus sector with all the ITER components inside, as shown in Fig. 2. It is a complex and large model, consisting of $\sim 5,000$ cells defined with over 3,050 surfaces. Its radius is $17 \mathrm{~m}$ and it has a height of $25.5 \mathrm{~m}$. The neutron source was modeled as pointwise source with spatial and 


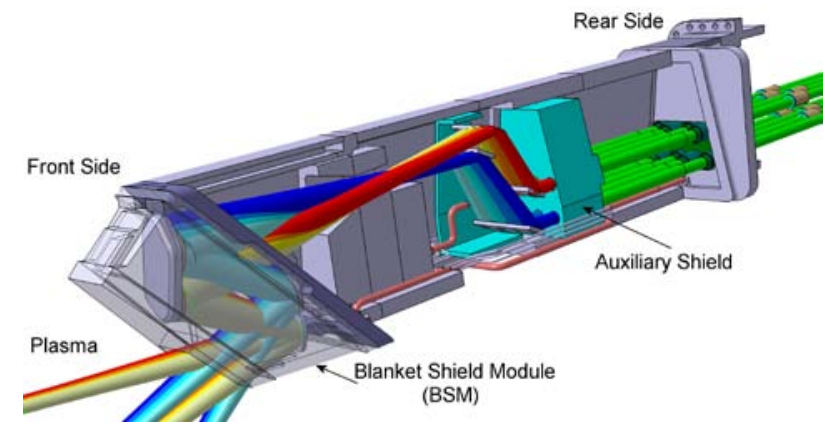

Fig. 1 CAD model of the QO design of the ECH launcher to be installed in ITER upper port

energy (Gaussian $14 \mathrm{MeV}$ D-T fusion spectrum) distributions inside the volume of plasma chamber shown in Fig. 2. Geometry of the ECH launcher was converted from CAD files into the MCNP5 input deck using McCad. After that dummy shield plug inside the ITER upper port was replaced with the ECH launcher as depicted in Fig. 2. The challenges of the ECH launcher integration into the Alite ITER model, as well as an overview of neutronic characteristics of the launcher and evolution of its shielding computations have been addressed elsewhere. ${ }^{7-9)}$ The need for detailed neutronic modeling of the QO design of the ECH launcher requires substantial computational resources which were satisfied by means of utilization of HPC-FF supercomputer described in Section II.

\section{MCNP5 Parallel Performance}

Because the MC radiation transport simulations reproduce random particle tracks independently, it is possible to compute the particle histories on parallel multiprocessor systems. The MCNP5 code has been installed on the High Performance Computer dedicated For Fusion applications (HPC-FF). It is based on petaflop architectures of JUROPA/HPC-FF system currently in operation at Juelich Supercomputing Centre (JSC), Research Centre Juelich (FZJ) in Germany. ${ }^{10)}$ Performance assessments of the MCNP5 code were carried out to find an efficient way to run the code in a parallel regime. The parallel version of the code has been compiled with the Intel Professional Fortran and $\mathrm{C} / \mathrm{C}++$ compilers, and uses the Message Passing Interface (MPI). In the computational scheme of the MPI-connected MCNP5 parallel job, the master processor sends the instructions to trace the nuclear reactions with each particle and to do the necessary computations to a selected number of slave CPUs. These instructions include the geometry definition and material composition. The MCNP5 parallel performance had been estimated previously ${ }^{11)}$ on 32 slave CPUs on the CampusGrid Linux cluster at FZK, reorganized into the Opus ${ }^{\mathrm{B}}$ cluster at KIT composed of the Opteron processor nodes connected by the InfiniBand (IB) switching fabric of network topology under the Scientific Linux 4.x operating system. The computational experience $^{11)}$ gained on the CampusGrid has been considered during the deployment of the MCNP5 parallel jobs on HPC-FF. ${ }^{12)}$ Practice of MCNP5 MPI-parallel computa-

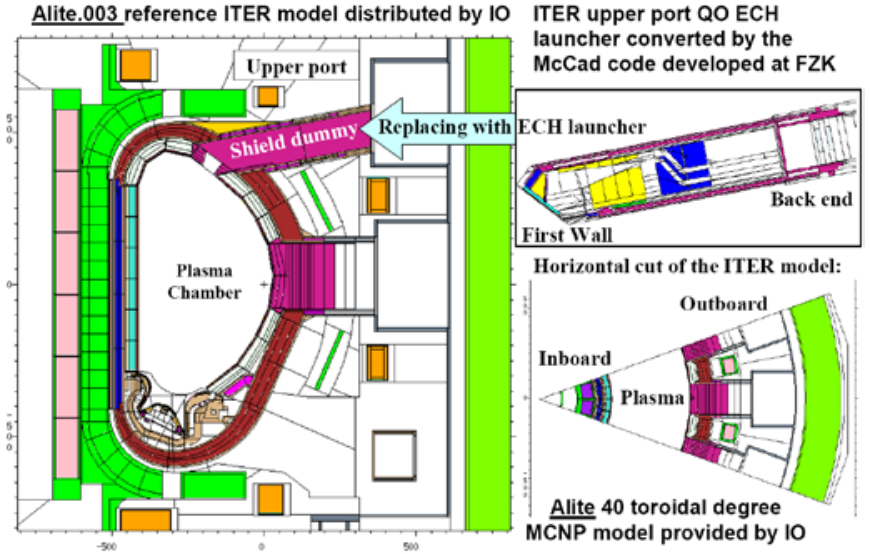

Fig. 2 Incorporation of the McCad converted QO ECH launcher model inside the Alite.003 standard ITER model, with its vertical (on the left and right upper sides) and horizontal (right bottom side) cuts

tions $^{11,12)}$ shows that it is important to keep the master-slave communication as little as possible. This is achieved by setting the number of intermediate data exchange, called "rendezvous", to a minimum using the PRDMP card of MCNP5. The optimal number of CPUs used in MCNP5 parallel calculations is dependent on complexity of the model, physical process involved in particle track and particle history longevity. As it was found, ${ }^{11,12)}$ the optimal number of CPUs is dependent on the MCNP5 job size expressed in number of histories set by the NPS card.

The results of the parallel performance and search for optimal number of CPUs are presented here in assumption of MCNP5 parallel run without intermediate rendezvous. Values in the PRDMP card are equal to total number of histories set by NPS. On the HPC-FF cluster there are 1,080 compute nodes with 8 cores $(8,640 \mathrm{CPU}$ cores in total) intended for batch parallel jobs. Each node includes 2 Intel Nehalem-EP quad-core processors (Xeon X5570 at $2.93 \mathrm{GHz}$ ) and $24 \mathrm{~GB}$ memory. The nodes are connected by means of the InfiniBand Mellanox ConnectX QDR HCA network adapter cards and multiple-port switches in the InfiniBand topology. ${ }^{10)}$ The operating system on HPC-FF is Linux SUSE SLES 11. The batch system is MOAB with underlying resource manager TORQUE. MOAB is a workload manager product of Cluster Resources, Inc. (www.clusterresources.com). The MOAB scheduler provides a utility to set polices for fair utilization of the available computational resources. By means of the MOAB scheduler commands, a user can submit parallel jobs and monitor their execution. When user sets certain number of CPUs in the MOAB script, then the NPS number is divided by this CPU number minus one. This is because in MPI-scheme one CPU is reserved for master process to control partial MCNP jobs running on the slave CPUs. And these portions of particle histories are computed on the slave CPUs until termination by limit of elapsed computation time or by number of processed histories. After that the MCNP tally results are collected from the slaves to form the output data. This was a very brief description on the operational level of the MCNP5 MPI- parallelism, but on practice, the 


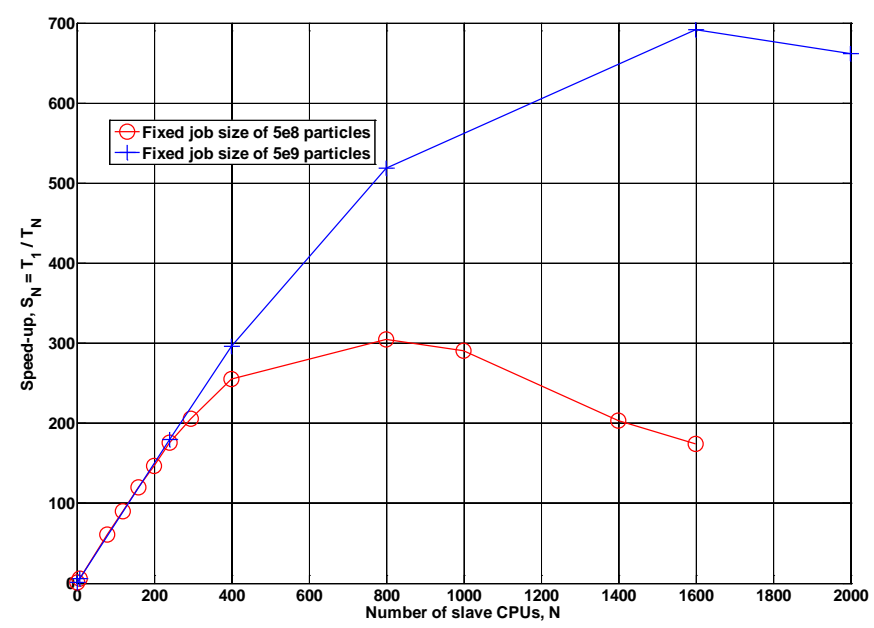

Fig. 3 Speedup for two fixed MCNP5 parallel job sizes of 5e8 and $5 \mathrm{e} 9$ particles sampled on HPC-FF

performance of the parallel run is decreased by the time spending for communications.

It is possible to distinguish two types of communications for the MPI parallel runs on a cluster: (1) MPI communications between compute nodes; (2) Input/Output (IO) data transfers between the compute nodes and data storage. On the HPC-FF system, the MPI-communication are supported by the state-of-the-art ParaStation MPI version 5.0 protocols, see details on the web: http://www.par-tec.com/products /parastation-mpi.html. The parallel file-system employed on HFC-FF is Lustre storage pool, ${ }^{10)}$ which is optimized for large IO data transfers. The IO performance is important for the large-scale parallel jobs. One indicative IO parameter is available bandwidth of the system, in case of Lustre on HPC-FF, the aggregated bandwidth is $9 \mathrm{~GB} / \mathrm{s}$ stripped over 56 Object Storage Targets (OSTs) on its \$WORK file-system recommended for large files and high performance requirements. At the MCNP job initiation, the master CPU sends copies of the 3D MCNP geometry description with the nuclear cross-section data in a binary format file "RUNTPE". By the end of the MCNP run, the partial tally results produced by each slave CPU should be sent back to the data storage and collected together into the resulted "RUNTPE" file and several types of the MCNP output files depending on the user settings. Therefore, along the MCNP MPI-execution, the code must run in serial sequence at least two times (if the intermediate rendezvous were excluded): at the beginning to deploy the job on the slave compute nodes and by the end to collect and process the tally results. The time spending for these serial fractions of the MCNP parallel run reduces the parallel performance. Information about the sizes of data to be distributed and collected back can be gathered by the sizes of the dumped information stored in the "RUNTPE" binary file. For the particular MCNP job of nuclear heating calculation using mm-scale mesh-tally technique which is described in Section III. 1, the size of initial "RUNTPE" file was 643.4 MB; it includes geometry and cross-sections data. It was produced by set no tallies in the

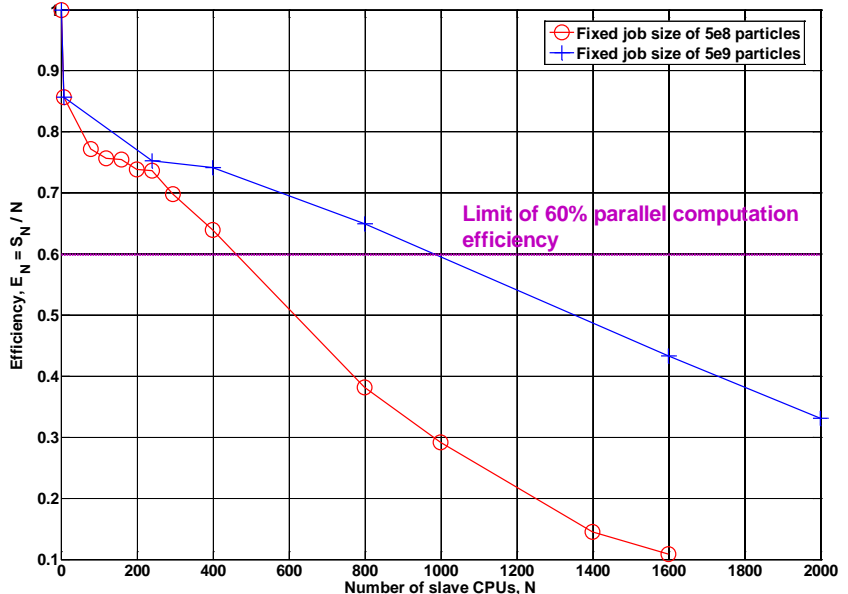

Fig. 4 Efficiency for two fixed MCNP5 parallel job sizes of 5e8 and $5 \mathrm{e} 9$ particles sampled on HPC-FF

MCNP run and its size indicates the size of data to be distributed among the slave CPUs. The data size to be collected back to master CPU is inferred from the size of final "RUNTPE" file with all requested MCNP tally results, including mesh-tally. This file size was 712.4 MB. Comparing with the initial size of the "RUNTPE" file, it follows that tally results occupy only $10 \%$ of the data to be collected from the computational nodes, and main constituents of the data are cross-section and very complicated geometry description of the ITER machine.

To do the MCNP5 parallel performance analysis, computational wall-clock time has been measured in dependence of number of utilized slave CPUs and size of the job. The measurement results are presented in Figs. $\mathbf{3}$ and $\mathbf{4}$ in terms of speedup and efficiency respectively.

The speedup $\left(\mathrm{S}_{\mathrm{N}}\right)$ is defined as the ratio between the wall-time running on one CPU $\left(T_{1}\right)$ and the wall-time running on $N$ slave CPUs $\left(T_{N}\right), S_{N}=T_{1} / T_{N}$. The efficiency $\left(E_{N}\right)$ is estimated by the ratio between the speedup $\left(\mathrm{S}_{\mathrm{N}}\right)$ and the number of slave CPUs (N).

The bigger the job-size, the bigger the chunks of information are which will be processed by a single slave CPU, and the less the communications are between the master and the slave CPUs. Following Fig. 3 the speedup peak and the number of utilized CPUs are shifted to higher values when increasing the number of sampled particles from 5e8 to $5 \mathrm{e} 9$.

The MCNP5 parallel efficiency, plotted in Fig. 4, shows the same positive tendency of the job size increasing if many hundreds of CPUs are available. This plot allows judging a job from the comparison with a computational efficiency limit. In this case a limit of $60 \%$ was chosen as a good compromise between performance and availability of CPUs. The job size of 5e9 particles falls below this efficiency limit at about 1,000 slave CPUs. That is, using more CPUs is getting less effective, and according to Fig. 3 it even spends more time to run the job if more than 1,600 CPUs are used. The whole wall-clock time for the accomplishment of this $1,600-\mathrm{CPU}$ job of 5e9 sampled particles is 132 minutes. 


\section{Fusion Neutronics Applications in ITER Upper QO ECH Launcher}

Here in Section III, we present new results of specific neutronic tasks of the design development of the ITER upper Electron Cyclotron Heating (ECH) launcher which are selected by the criterion of high power of the MCNP5 parallel computations required to resolve neutronic heterogeneous effects. Considering the methodological aspects of this work, the MCNP5 code inherent features such as its variance reduction techniques (VRTs), mesh tallies, and use of MPI-parallelism have been enhanced and completed with the CAD-based radiation transport capabilities of the McCad geometry interface. Among the great variety of the VRTs of MCNP5, ${ }^{1)}$ in this work its weight window generator (WWG) has been used predominantly. The iterative use of WWG allows keeping the population of particles in tasks of radiation deep-penetration by means of automatic generation of weight window importance functions.

The neutronics results were normalized to $500 \mathrm{MW}$ of DT fusion power, $80 \%$ of which is released in form of $14 \mathrm{MeV}$ neutrons emitted from ITER plasma chamber depicted in Fig. 2, where cross-sections of the applied 3D Alite MCNP5 model are plotted.

As an example of success in the accomplishment of the highly demanding task, high-resolution mapping of the helium production distribution in the ITER location supposed to be re-welded was established using the MCNP mesh-tally. ${ }^{9,12)}$ It was important to calculate this mapping because the re-weldability of irradiated steel is limited by the content of generated helium. Use of the HPC-FF system gave the possibility to obtain the MCNP5 results with a high-resolution $2 \times 2 \times 2 \mathrm{~mm}^{3}$ mesh tally. The mesh tally grid superimposed over complex launcher geometry revealed neutronic heterogenic effects in helium gas production rate in the corner of Vacuum Vessel and ITER upper ECH launcher. The analysis of mesh-tally calculations has revealed the locations of an extensive helium production up to 19 appm per 0.63 full power year in the boron-doped ferromagnetic steel of SS304B4 grade, which exceeds the helium content allowed for re-weldability by a factor of 19. This outcome excludes the possibility to re-weld these ferromagnetic plates inserted in the structure of ITER Vacuum Vessel (VV) and influences the shielding arrangement. ${ }^{9,12)}$

\section{Nuclear Heating Distribution in Blanket Shield Module}

Nuclear heating is a key input parameter for structural analyses of the launcher and adjacent components in ITER and it plays an important role in designing of the reactor components to be exposed to neutron radiation in ITER. Neutron radiation causes production of secondary photons which interact with matter, and these photons induce heat deposition which becomes predominant in heavy (high atomic mass) materials such as steel or copper used in structures of the series of First Wall Panels layers (FWP-1-2-3) of the Blanket Shield Module (BSM) at the front side of the ITER ECH launcher shown in Fig. 1. The spatial averaged nuclear heating results for BSM with separation of neutron
Table 1 MCNP cell-averaged nuclear heating in FWP and BSM

\begin{tabular}{cccc} 
Position & $\begin{array}{c}\text { Neutron (n) } \\
\text { heating, W/cm }\end{array}$ & $\begin{array}{c}\text { Photon (p) } \\
\text { heating, W/cm }\end{array}$ & $\begin{array}{c}\text { Total } \mathrm{n}+\mathrm{p} \\
\text { heating, } \mathrm{W} / \mathrm{cm}^{3}\end{array}$ \\
\hline FWP1: Be & $2.47 \mathrm{E}+00$ & $5.57 \mathrm{E}-01$ & $3.03 \mathrm{E}+00$ \\
\hline $\begin{array}{c}\text { FWP2: Copper } \\
\text { heat sink }\end{array}$ & $1.09 \mathrm{E}+00$ & $3.47 \mathrm{E}+00$ & $4.56 \mathrm{E}+00$ \\
\hline $\begin{array}{c}\text { FWP3: Copper } \\
\text { heat sink }\end{array}$ & $9.24 \mathrm{E}-01$ & $3.39 \mathrm{E}+00$ & $4.31 \mathrm{E}+00$ \\
\hline $\begin{array}{c}\text { BSM - shield } \\
\text { block }\end{array}$ & $1.56 \mathrm{E}-01$ & $8.11 \mathrm{E}-01$ & $9.67 \mathrm{E}-01$ \\
\hline
\end{tabular}

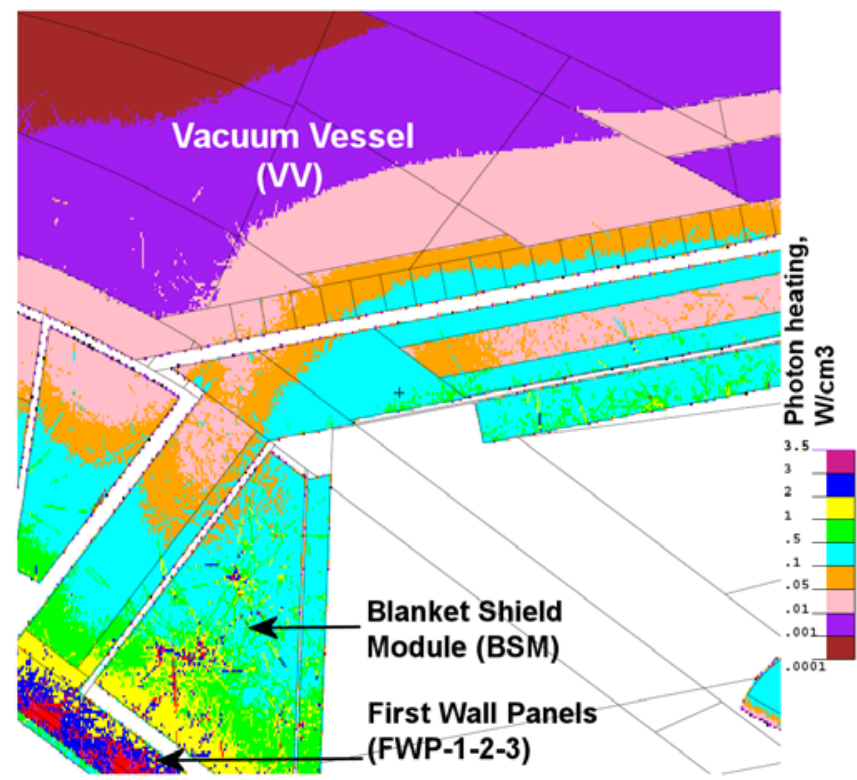

Fig. 5 Map of photon heating distribution, $\mathrm{W} / \mathrm{cm}^{3}$

and photon deposition are presented in Table 1. Obviously, neutron deposition dominates heating in light material (beryllium), and photon heating dominates in heavy materials, making maximum of $4.56 \mathrm{~W} / \mathrm{cm}^{3}$ in total heat on the second FWP2 layer of copper heat sink. The map of photon heating is depicted in Fig. 5 using fine $2 \times 2 \times 2 \mathrm{~mm}^{3}$ mesh tally, and the results are consistent with Table 1 .

Figure 6 shows a color map of the MCNP5 statistical relative error $(\mathrm{R})$ given at one standard deviation $(1 \sigma)$ within $68 \%$ confidence interval of the photon heating results. As recommended, ${ }^{1)}$ a result calculated with the track-length estimation tally of MCNP is generally reliable if the associated statistical error $\mathrm{R}$ is less than 0.10 . This is true for most part of BSM and VV being analysed. A tally result is questionable if $0.1<\mathrm{R}<0.2$, and it could differ by a factor of a few if $0.2<\mathrm{R}<0.5$. The error estimation indicates about general reliability for the results obtained around the critical location. Visual non-uniformity in Figs. 5 and 6 such as dash-lines inside the BSM and Vacuum Vessel is explained by statistical fluctuation of particles, and it is found in particle low-populated locations in middle of BSM and in-depth of VV. Because at these non-uniformity locations errors are more than $20 \%-30 \%$, the results in these exceptional locations should be not used. 


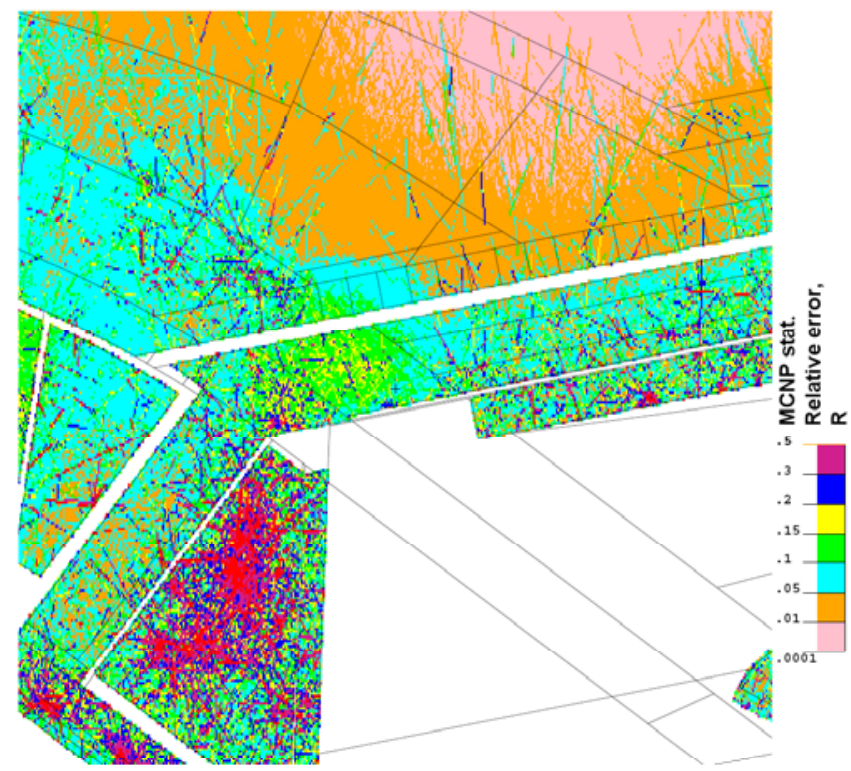

Fig. 6 MCNP5 statistical relative errors of photon heating

\section{Nuclear Heating Analysis for Auxiliary Shield}

The auxiliary shield blocks have been arranged in the center of the ECH QO launcher serving for radiation shielding and bearing structure support functions. They are positioned behind the mm-wave optics mirror system and composed with three shield blocks made of solid 100\% SS 316L(N)-IG ITER grade steel without any cooling substances such as water or helium gas. The mass density of steel is $7.93 \mathrm{~g} / \mathrm{cm}^{3}$. The CATIA model of the auxiliary shield blocks was converted by the McCad geometry interface into the MCNP5 representation and then incorporated inside the upper port launcher of the Alite.003 standard ITER model as shown in Fig. 7. Then the MCNP5 3D mesh-tally was defined and superimposed over the auxiliary shield and upper port walls around it. The mesh-tally grid is plotted in Fig. 7 and the nuclear heating results calculated on it are mapped using the standard MCNP5 plotter in Figs. 8 and 9. The results are normalized on $500 \mathrm{MW}$ of ITER fusion power. The heating has been calculated in the low-resolution mesh-tally with its cell size of $8 \times 8 \times 8 \mathrm{~cm}^{3}$. This size was chosen because the spatial-averaged heating was very small at $\sim 2 \mathrm{~m}$ distance outside from the plasma.

The space-averaged nuclear heating results calculated for the three blocks of the auxiliary shield are listed in Table 2, where photon and neutron heat deposits are presented separately and also as a sum. As followed from Table 2, the main contribution to nuclear heating is produced by the photon heat deposition. According to the spatial-averaged nuclear heating results of Table 2, the photon heating is higher than the neutron heating by the factor in a range of 32-56, which means the photon heating dominates in nuclear heating, generating $97 \%$ - 98\% of total heating. For this reason, the maps of nuclear heating distribution are plotted mostly for photon heat deposition, e.g. in Fig. 8 photon map is shown on the 3D mesh-tally central cut by perpendicular to y-axis plane at $\mathrm{y}=0 \mathrm{~cm}$. Figure 9 shows a map orthogonal to Fig. 8 along its A2 cut pointed out in Fig. 8.

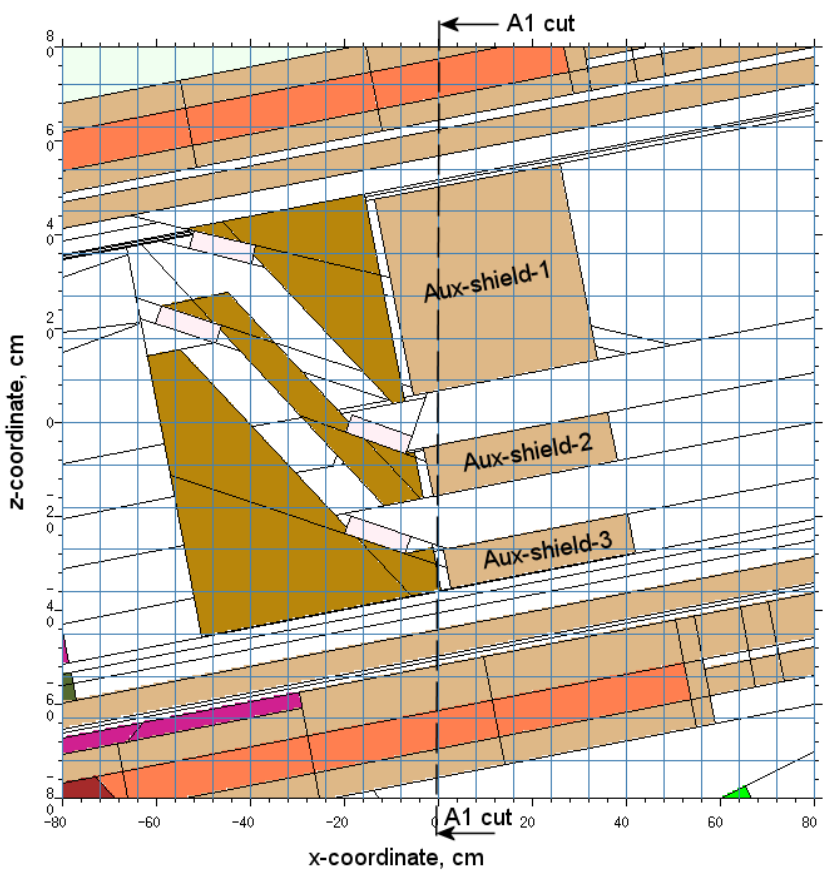

Fig. 7 Vertical radial-poloidal (x-z) cut by central plane at $\mathrm{y}=0 \mathrm{~cm}$ of the Alite.003 MCNP model with inserted ECH launcher. The mid-part of the launcher with superimposed mesh-tally is cut through the three auxiliary shield blocks (Aux-shield-1-2-3).

Table 2 Nuclear heat density $\left(\mathrm{W} / \mathrm{cm}^{3}\right)$ in spatially-averaged over the MCNP5 model cells of the auxiliary shield blocks

\begin{tabular}{|c|c|c|c|c|}
\hline $\begin{array}{c}\text { Auxiliary } \\
\text { shield } \\
\text { block }\end{array}$ & $\begin{array}{c}\text { Shield } \\
\text { block } \\
\text { volume, } \\
\mathrm{cm}^{3}\end{array}$ & $\begin{array}{l}\text { Photon } \\
\text { heating, } \\
\text { W/ } \mathrm{cm}^{3}\end{array}$ & $\begin{array}{l}\text { Neutron } \\
\text { heating, } \\
\mathrm{W} / \mathrm{cm}^{3}\end{array}$ & $\begin{array}{c}\text { Sum heat- } \\
\text { ing (photon } \\
+ \text { neutron), } \\
\mathrm{W} / \mathrm{cm}^{3}\end{array}$ \\
\hline $\begin{array}{c}\text { Aux- } \\
\text { shield-1 }\end{array}$ & $6.79 \mathrm{E}+04$ & 7.60E-05 & $2.40 \mathrm{E}-06$ & 7.84E-05 \\
\hline $\begin{array}{c}\text { Aux- } \\
\text { shield-2 }\end{array}$ & $1.67 \mathrm{E}+04$ & 8.81E-05 & $1.58 \mathrm{E}-06$ & 8.96E-05 \\
\hline $\begin{array}{c}\text { Aux- } \\
\text { shield-3 }\end{array}$ & $1.36 \mathrm{E}+04$ & 8.87E-05 & $2.00 \mathrm{E}-06$ & 9.07E-05 \\
\hline
\end{tabular}

It is observed in Fig. 8 that heating reaches local maximal values at the upper part of the auxiliary shield block-1, and in order to find this maximum more precisely, additional A2 cut was arranged. The resulting photon heating distribution along the A2 cut is presented in Fig. 9, where heating in the block-1 is limited by $5 \mathrm{e}-4 \mathrm{~W} / \mathrm{cm}^{3}$. The location of heat-peaking area is confirmed by more sophisticated 3D maps getting available by means of using VisIt 2.0.2 graphics tool, ${ }^{13)}$ which certainly could enhance capabilities of MCNP to plot mesh-tally results in 3D maps. The VisIt tool is software developed by the Department of Energy (DOE) Advanced Simulation and Computing Initiative (ASCI) to visualize and analyze the results of terascale simulations. Figure 10 illustrates sum of photon and neutron heating distribution on 3D mesh-tally map with pseudo-color scale. Because the Visit tool allows plotting maps using only ASCII text format of mesh-tally results, a variety of mathematical operations are allowed for post-processing of the heating results. 


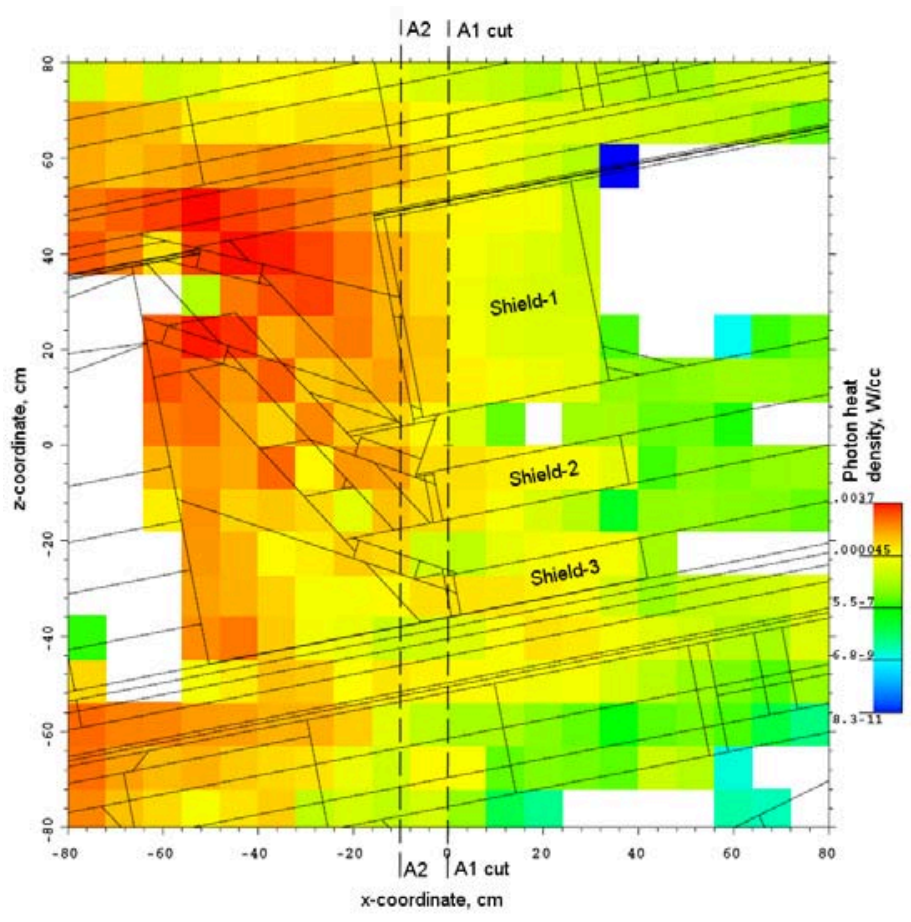

Fig. 8 Map of photon heat density $\left(\mathrm{W} / \mathrm{cm}^{3}\right)$ superimposed over the vertical radial-poloidal (x-z) cut of the MCNP mesh-tally depicted in Fig. 7

The fragmentation of heating distributions maps into multicolor mosaic on the edges of the materials is explained by the misalignment between the arbitrary oriented MCNP cells and the regular $\mathrm{x}-\mathrm{y}-\mathrm{z}$ rectangular cells of mesh-tally used for heating calculations. The heating is averaged inside the mesh cells. Hence if going along the border regions between material and void, the material-to-void ratio inside the mesh-tally elements is changing substantially, causing quite visible difference in averaged mesh-tally heating shown in Figs. 8-10. This difference can be diminished by reducing the size of mesh-tally cells. Practically, the MCNP5 mesh tally technique was applied for heating calculations by using the FMESH card with its FM tally multiplier card in which the material number " $m$ " was set to zero, implying that the heating cross sections for the material in which the particle is traveling are used.

\section{Conclusion}

It is demonstrated in this paper that for the ITER heterogeneous models with a possibility of radiation streaming effects resulting in hot-spots, precise 3D mapping of the results obtained in geometry model close to the original CAD model is required. The statistical errors associated with the mesh-tally results were reduced by applying VRTs and by taking the advantages of the MCNP5 MPI parallel computations on the JUROPA/HPC-FF High Performance Computer system operated by the Juelich Supercomputer Centre (JSC) at FZJ. The presented examples of the MCNP5 parallel calculations for the design support of the ECH upper launcher have proved that certain types of neutronic calculations

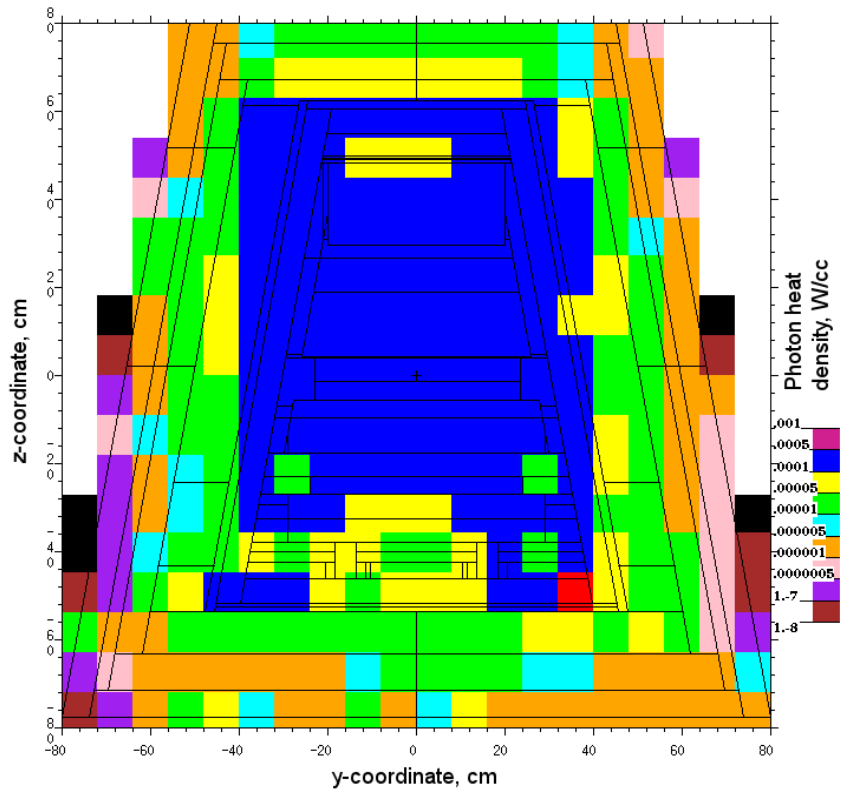

Fig. 9 Map of photon heat density $\left(\mathrm{W} / \mathrm{cm}^{3}\right)$ superimposed over the vertical toroidal-poloidal ( $\mathrm{y}-\mathrm{z})$ A2 cut MCNP mesh-tally depicted in Fig. 8. Mosaic picture is caused by misalignment between the MCNP geometry and the regular mesh-tally cells.

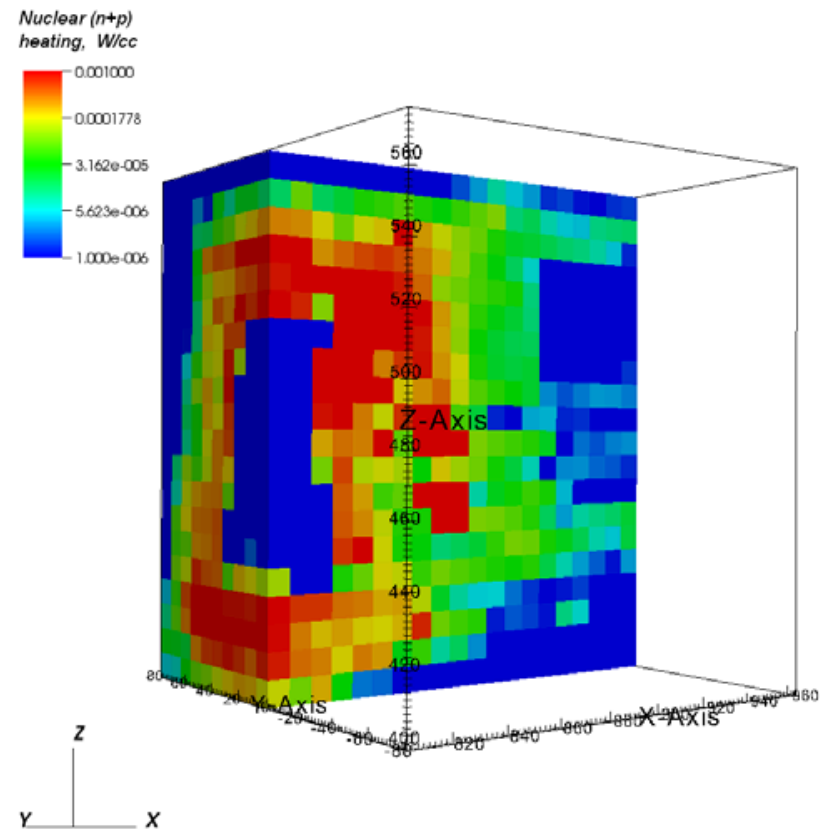

Fig. $103 \mathrm{D}$ map of total nuclear heating $\left(\mathrm{W} / \mathrm{cm}^{3}\right)$ with photon and neutron heat depositions. Mosaic picture is caused by misalignment between the MCNP geometry and the regular mesh-tally cells.

could be done only on supercomputers. Such radiation transport implementation supported by CAD-based automatic model converters and 3D visualizing tools (e.g. McCad and VisIt) preserves geometry complexity, reduces error-prone human involvement in the MCNP5 geometry modeling, and speeds up iterative design process of the ITER components development. 
The spatial distributions of nuclear heating have been analysed by using the graphical representation of the MCNP5 mesh-tally results in 2D and 3D plots. High heating of the launcher Blanket Shield Module (BSM) reaching $4.56 \mathrm{~W} / \mathrm{cm}^{3}$ imposes essential requirement on the cooling system design with effective heat-removal. From the nuclear heating analysis in the auxiliary shield of the ITER upper QO ECH launcher, using the MCNP5 mesh-tally spatial distribution, it is possible to conclude that peak nuclear heating in the auxiliary shield blocks is limited by $5 \mathrm{e}-4 \mathrm{~W} / \mathrm{cm}^{3}$. This low value indicates that no additional active cooling is required. The cell size of the used mesh tally was defined by the structural designing needs requested for nuclear heating estimations. In case of high heating inside BSM the mesh tally resolution was very fine (on mm-scale), while low heating inside the auxiliary shield was calculated on low-resolution grid of 8-cm scale.

Other examples of the use of the HPC-FF computer system for the MCNP5 parallel applications to neutronic analyses for several different ITER components could be found elsewhere. ${ }^{14)}$

\section{Acknowledgment}

This work, supported by the European Communities under the contract of Association between EURATOM and Karlsruhe Institute of Technology, was carried out partly within the framework of the European Fusion Development Agreement and partly under contractual arrangements with the Fusion for Energy Joint Undertaking (F4E). Views and opinions expressed herein do not necessarily reflect those of the European Commission or those of F4E. This work was carried out using an adaptation of the Alite MCNP model which was developed as a collaborative effort between the FDS team of ASIPP China, ENEA Frascati, JAEA Naka, CCFE and the ITER Organization.

\section{References}

1) X-5 Monte Carlo Team, MCNP - A General Monte Carlo $N$-Particle Transport Code, Version 5, Volume I, MCNP Overview and Theory, LA-UR-03-1987 (Revised 10/3/05), Los Alamos National Laboratory (LANL) (2003).
2) D. Grosse, H. Tsige-Tamirat, "Current Status of the CAD Interface Program McCad for MC Particle Transport Calculations,” Proc. M\&C 2009, Saratoga Springs, New York, May 3-7, 2009, American Nuclear Society (2009), [CD-ROM].

3) A. Serikov et al., "Use of McCad for the generation of MCNP models in fusion neutronics," Proc. M\&C 2009, Saratoga Springs, New York, May 3-7, 2009, American Nuclear Society (2009), [CD-ROM].

4) A. Serikov et al., "Nuclear-Safety-Related and Shielding Analyses of the ITER Quasi-Optical ECH Launcher," IEEE Trans. Plasma Science, 38[3], 224-231 (2010); http://dx.doi.org/10.11090TPS.2009.2032261

5) R. Heidinger et al., "Conceptual design of the ECH upper launcher system for ITER,” Fusion Eng. Des., 84, 284-289 (2009); http://dx.doi.org/10.1016/j.fusengdes.2008.11.002

6) M. J. Loughlin, et al., "ITER nuclear analysis strategy and requirements,” Fusion Sci. Technol., 56[2], 566-572 (2009).

7) A. Serikov, U. Fischer et al., "Nuclear analyses for the ITER ECH launcher,” Nucl. Fusion, 48, 054016 (2008); http://dx.doi.org/10.1088/0029-5515/48/5/054016.

8) A. Serikov et al., "Overview of recent nuclear analyses for the Upper ECH launcher in ITER,” Fusion Eng. Des., (2010); doi:10.1016/j.fusengdes.2010.06.016

9) A. Serikov et al., "Evolution of shielding computations for the ITER upper ECH launcher," Proc. of the ANS RPSD 2010 Joint Topical Meeting, Las Vegas, NV, April 18-23 (2010), Nucl. Technol., in press, corrected proof, 175 (July 2011).

10) HPC-FF system: http://www2.fz-juelich.de/jsc/juropa/, and http://www2.fz-juelich.de/jsc/juropa/configuration/

11) A. Serikov et al., "Performance assessments of the MCNP5 parallel computations for the ITER ECRH launcher," Proc. German Ann. Meet. on Nuclear Technology (Jahrestagung Kerntechnik JK-2008), May 27-29, 2008, Hamburg, Germany, (2008), [CD-ROM].

12) A. Serikov et al., "MCNP5 parallel computations on JUROPA/HPC-FF supercomputer for ITER applications,” Proc. German Annual Meeting on Nuclear Technology (Jahrestagung Kerntechnik JK-2010), May 4-6, 2010, Berlin, Germany, (2010), [CD-ROM].

13) VisIt Tool: https://wci.llnl.gov/codes/visit/home.html

14) A. Serikov et al., "Toolkit for High Performance Monte Carlo Radiation Transport and Activation Calculations for Shielding Applications in ITER,” Proc. M\&C 2011, Rio de Janeiro, RJ, Brazil, May 8-12, 2011, LAS/ANS (2011), [CD-ROM]. 